\title{
Perfil clínico e terapêutico dos pacientes com hipertireoidismo do ambulatório de endocrinologia de um hospital universitário do sul do Brasil
}

\author{
Clinical and therapeutic profile of patients with hyperthyroidism in an outpatient \\ endocrine clinic at a university hospital in southern Brazil \\ Rafael Antonio Parabocz ${ }^{1}$ (D), Renata Soares Carvalho ${ }^{1}$ (D), Gianna Carla Alberti Schrut ${ }^{1}$ (D), Ana Claudia \\ Garabeli Cavalli Kluthcovsky ${ }^{1 \mathbb{D}}$, Matheo Augusto Morandi Stumpf ${ }^{1}$
}

\begin{abstract}
RESUMO
Introdução: O hipertireoidismo decorre da elevação sérica dos hormônios tireoidianos, secundária à hiperfunção da glândula tireoide, sendo as principais causas a Doença de Graves (DG) e os Bócios Nodulares Tóxicos (BNT). Objetivos: Avaliar o perfil clínico e modalidades terapêuticas aplicadas em pacientes com diagnóstico de hipertireoidismo acompanhados em um Hospital Universitário no Sul do Brasil. Métodos: Estudo observacional, transversal e descritivo, com inclusão de pacientes com diagnóstico de hipertireoidismo por DG e BNT; foram coletados dados epidemiológicos, clínicos, laboratoriais e de tratamento. Resultados: A maioria dos pacientes foi referenciada pela atenção primária e encontrava-se em uso prévio de droga antitireoidiana (DAT). A variável idade obteve diferença estatisticamente significativa entre as etiologias de DG e BNT; em ambas houve predomínio de incidência no sexo feminino. A DG apresentou maior frequência de sinais e sintomas de tireotoxicose, ao passo que o BNT mostrou mais sinais e sintomas de compressão. Houve remissão da doença em $23,2 \%$ dos pacientes com DG tratados com DAT; em $23,2 \%$ dos pacientes optou-se pela manutenção de metimazol em baixa dose por mais de 36 meses e em $16,1 \%$ foi realizado tratamento definitivo. No BNT foram preferidas terapias definitivas, principalmente a tireoidectomia, em 27,5\% dos pacientes. Doses baixas de metimazol por mais de 36 meses foram utilizados também no BNT, em 22,5\% dos pacientes. Conclusão: O hipertireoidismo é uma doença heterogênea, desde a clínica inicial até a terapêutica, entre suas etiologias mais prevalentes. Observou-se uma tendência de priorizar as terapias medicamentosas em longo prazo com baixas doses, tanto na DG quanto no BNT.
\end{abstract}

Palavras-chave: Hipertireoidismo, Doença de Graves, Bócio nodular.

\begin{abstract}
Introduction: Hyperthyroidism results from the serum elevation of thyroid hormones, secondary to hyperfunction of the thyroid gland. The main causes are Graves' disease (DG) and Toxic Nodular Goiters (BNT). Objectives: Evaluate the clinical profile and therapeutic modalities applied in patients diagnosed with hyperthyroidism followed up at a University Hospital in Southern Brazil. Methods: Observational, cross-sectional and descriptive study, including patients diagnosed with hyperthyroidism by DG and BNT; epidemiological, clinical, laboratory and treatment data were collected. Results: Most patients were referred by primary care and had been using antithyroid drugs (DAT). The age variable obtained showed a statistically significant difference between the etiologies of DG and BNT; in both, there was a predominance of incidence in females. DG showed a higher frequency of signs and symptoms of thyrotoxicosis, while BNT showed more signs and symptoms of compression. There was remission of the disease in $23.2 \%$ of patients with DG treated with DAT; in $23.2 \%$ of the patients, low-dose methimazole was maintained for more than 36 months and in 16.1\%, definitive treatment was performed. In BNT, definitive therapies were preferred, mainly thyroidectomy, in $27.5 \%$ of patients. Low doses of methimazole for more than 36 months were also used in BNT in $22.5 \%$ of patients. Conclusion: Hyperthyroidism is a heterogeneous disease, from initial clinic to therapy, among its most prevalent etiologies. There was a tendency to prioritize long-term drug therapies with low doses, both in DG and BNT.
\end{abstract}

Keywords: Hyperthyroidism, Graves' disease, Nodular goiter.

1. Universidade Estadual de Ponta Grossa - UEPG, (PR), Brasil. 


\section{INTRODUÇÃO}

A tireotoxicose é uma síndrome clínica hipermetabólica, decorrente da elevação dos níveis de hormônios tireoidianos circulantes: tiroxina (T4) e triiodotironina (T3). Quando esse aumento é secundário a um estado de hiperfunção da glândula tireoide, dá-se o nome de hipertireoidismo ${ }^{1,2}$. Os dois termos, hipertireoidismo e tireotoxicose, são usualmente empregados de forma intercambiável ${ }^{1}$.

O hipertireoidismo é uma desordem endócrina comum, afetando $1 \%$ a $1,5 \%$ da população. A prevalência aumenta com a idade e atinge com mais frequência o sexo feminino. Dentre as causas de hipertireoidismo mais prevalentes estão a doença de Graves (DG), que representa até $80 \%$ dos casos, e os bócios nodulares tóxicos (BNT) - uni ou multinodular. A etiologia é multifatorial; participam fatores genéticos e ambientais, como a deficiência do aporte de iodo na dieta e o tabagismo ${ }^{3}$.

O hipertireoidismo por DG é o resultado da hiperplasia difusa do tecido tireoidiano ${ }^{2}$. Ocorre devido à atividade de autoanticorpos, que ocupam os receptores de TSH e exercem atividade estimuladora sobre eles, elevando a produção de T3 e T4 por todo o parênquima folicular. Esses anticorpos atuam de forma sistêmica, depositando-se em outras regiões do corpo, como a órbita - gerando oftalmopatia - e menos comumente a pele - caracterizando a dermopatia ${ }^{1}$. Os BNT podem ser de dois tipos: bócio multinodular tóxico (BMNT) ou bócio uninodular tóxico (BUNT). O BMNT é a evolução de um bócio multinodular não tóxico, quando alguns desses nódulos adquirem autonomia funcional, tornandose produtores de hormônios tireoidianos ${ }^{3}$. O BUNT é mais raro que o BMNT; trata-se de um tumor benigno nodular único, com capacidade de crescer - geralmente maior que $3 \mathrm{~cm}$ - e produzir T3 e T4 de forma autônoma. O BUNT também pode ser denominado adenoma tóxico ou doença de Plummer ${ }^{4}$.

O diagnóstico laboratorial de hipertireoidismo é realizado pelos níveis séricos do hormônio tireoestimulante (TSH), T3 e T4 livre. O TSH é o exame mais sensível para estabelecer o diagnóstico de hipertireoidismo, e valores de TSH abaixo da referência confirmam a hipótese ${ }^{5}$.

Para diferenciar a etiologia do hipertireoidismo, podem ser realizadas as dosagens de anticorpos, cintilografia com taxa de captação de iodo e ultrassonografia de tireoide ${ }^{5}$. Na DG, os níveis de anticorpo anti-receptor de TSH (TRAb) estarão elevados, o que geralmente não ocorre nas doenças nodulares ${ }^{2}$. A confirmação da etiologia pode ser realizada pela taxa de captação de iodo radioativo pela cintilografia de tireoide. Na DG ocorre aumento na captação de iodo radioativo, com distribuição difusa ${ }^{5}$. No BMNT serão observadas múltiplas áreas focais (nodulares) de hipercaptação, com hipocaptação do restante da glândula. No BUNT é encontrado uma área focal única de hipercaptação, com supressão total ou parcial do parênquima tireoidiano restante ${ }^{4}$. A ultrassonografia quantifica o volume da tireoide e sua extensão cervical, e caracteriza as formações nodulares, algumas das quais poderão ter indicação de serem puncionadas, por punção aspirativa com agulha fina (PAAF), para se afastar o diagnóstico de malignidade, através do estudo citológico ${ }^{2,4}$.

O tratamento do hipertireoidismo pode ser realizado por três formas principais: drogas antitireoidianas (DAT), ablação do tecido tireoidiano com dose terapêutica de iodo radioativo (DTI ${ }^{131}$ ) ou tireoidectomia. Os dois últimos são considerados formas de tratamento definitivo ${ }^{5}$.

A DTI ${ }^{131}$ é usada como primeira linha no tratamento de DG na América do Norte ${ }^{2}$. No Brasil $^{5}$, está indicada apenas na DG com recidiva após tratamento com DAT, ou que necessitem de um controle rápido e definitivo. Para o BNT, orienta-se que a tireoidectomia seja considerada a primeira escolha de tratamento, na maior parte dos pacientes ${ }^{2,5}$.

No Brasil, as DAT são consideradas o tratamento de primeira linha para pacientes com DG. Também podem ser usadas no BNT, quando o paciente possui contraindicações à tireoidectomia. A droga de escolha é o metimazol, pela melhor tolerância e pela possibilidade de ser administrado em única dose diária. O propiltiouracil (PTU) é menos utilizado, devido aos relatos de hepatotoxicidade 5 . O metimazol apresenta poucos efeitos colaterais, como erupções cutâneas, intolerância gástrica e artralgia, que ocorrem em aproximadamente $5 \%$ dos pacientes. Eventos adversos graves são raros (menos de 0,5\%), representados pela agranulocitose ${ }^{6}$. Tanto os efeitos colaterais quanto os adversos estão associados a altas doses de DAT ${ }^{5-6}$.

Considerando-se a complexidade da sintomatologia, diagnóstico e principalmente a heterogeneidade de tratamento do hipertireoidismo 
em suas causas mais prevalentes, o presente estudo objetivou avaliar o perfil clínico e terapêutico de pacientes acompanhados por hipertireoidismo em um ambulatório de endocrinologia de um Hospital Universitário do Sul do Brasil.

\section{MÉTODOS}

Trata-se de um estudo observacional, transversal, descritivo e de abordagem quantitativa. Envolveu pacientes com registros de acompanhamento pelo Ambulatório de Endocrinologia do Hospital Universitário Regional dos Campos Gerais (HURCG), da cidade de Ponta Grossa, Paraná, entre janeiro de 2014 a dezembro de 2018. Revisou-se prontuários físicos de consultas anteriores ao segundo semestre de 2014, e eletrônicos para registros a partir deste período. Os prontuários foram fornecidos pela Companhia de Tecnologia da Informação e Comunicação do Paraná (CELEPAR), selecionados por Classificação Internacional de Doenças (CID), através do código E050, referente a hipertireoidismo e tireotoxicose.

Foram incluídos no estudo pacientes com diagnóstico de hipertireoidismo por DG e BNT (BUNT ou BMNT), que possuíam um ou mais registros no ambulatório de endocrinologia geral do HURCG. Como critério de exclusão, estão os pacientes do ambulatório de endocrinologia obstétrica, pelo elevado número de gestantes com tireotoxicose transitória gestacional ${ }^{7}$ e os pacientes de etiologia indeterminada por insuficiência de dados.

A determinação da etiologia do hipertireoidismo foi confirmada pela análise dos níveis de TRAb, pela taxa de captação de iodo na cintilografia, dados específicos de ultrassonografia (presença de nódulo, bócio ou de alteração ao Doppler), ou somente por dados clínicos altamente sugestivos (presença de oftalmopatia, por exemplo, indicando DG).

Após aprovação do Comitê de Ética em Pesquisa da instituição, a coleta de dados foi realizada de maneira padronizada para obtenção das variáveis a serem estudadas.

Para avaliação dos dados epidemiológicos, foram utilizadas as variáveis: sexo, idade e procedência. Também foram obtidas as informações sobre a forma de referência do paciente à atenção especializada do hospital (Unidade Básica de Saúde ou encaminhamento interno) e o manejo terapêutico pregresso ao acompanhamento no ambulatório.
Com relação à história clínica do paciente, foram coletados os sintomas referidos e os sinais encontrados nos registros de exame físico. Os valores de peso corporal e frequência cardíaca (FC) foram coletados da primeira e da última consulta.

Com relação à análise laboratorial, foram coletados os dados da primeira e última consulta, sendo considerados os valores de referência do laboratório de análises clínicas do HURCG: TSH de $0,3 \mathrm{uUI} / \mathrm{L}$ a 4,2 uUI/L; T4 livre de 0,9 ng/dL a 1,7 ng/ dL; T3 total de $80 \mathrm{ng} / \mathrm{dL}$ a $200 \mathrm{ng} / \mathrm{dL}$; e TRAb abaixo de 1,75 UI/L. Exames de imagem foram analisados, incluindo ultrassonografia e cintilografia de tireoide, para auxílio na definição da etiologia.

Foram coletadas informações com relação ao desfecho e tratamento dos pacientes. Dos que permaneceram em acompanhamento, ou receberam alta, foram analisados os possíveis desfechos do hipertireoidismo: (1) remissão da doença; (2) uso de metimazol em dose baixa por longo tempo; (3) opção por tratamento definitivo (tireoidectomia e DTI ${ }^{131}$ ). Tomou-se nota também dos pacientes que abandonaram o ambulatório.

Nos pacientes com DG, foi considerado remissão o período mínimo de 12 meses em estado de eutireoidismo, na ausência de DAT. Foi considerada recidiva a presença de TSH suprimido e níveis elevados de T3 e/ou T4 séricos após tentativa de suspender medicação, associado ou não a sinais clínicos de hipertireoidismo.

O armazenamento dos dados foi feito no software Microsoft Office Excelß, versão 2010. As variáveis categóricas foram descritas através de distribuição de frequências absoluta e relativa. Quando contínuas, as variáveis foram expressas com medidas de tendência central, amplitude e desvio padrão. Para a análise estatística dos resultados, foi utilizado o programa Statistical Package for Social Science (IBM SPSS Statistics), versão 15.0. Os testes qui-quadrado e exato de Fisher foram escolhidos para determinar as associações e diferenças nos conjuntos de dados categóricos. Os testes de Wilcoxon e $t$ de Student para amostras pareadas foram usados para comparação das variáveis contínuas antropométricas, sinais vitais e laboratoriais (o primeiro quando a distribuição da amostra foi não paramétrica e o segundo para distribuição paramétrica). Em todos os testes o nível de significância foi fixado em 0,05 ou $5 \%$. 


\section{RESULTADOS}

Do total de 153 prontuários analisados, 57 foram excluídos pelos critérios de exclusão adotados pelo estudo. Os pacientes incluídos foram separados em dois grupos, de acordo com a etiologia do hipertireoidismo: DG e BNT (este, dividido em subgrupos: BMNT e BUNT). Portanto, 0 estudo analisou 96 prontuários, em dois grupos, por etiologia: 56 pacientes com DG e 40 pacientes com BNT (36 por BMNT e 4 por BUNT).

Dos 56 pacientes com DG, $38(67,86 \%)$ foram submetidos à ultrassonografia, que mostrou em 36 pacientes um aumento difuso do parênquima tireoidiano; $22(39,28 \%)$ à cintilografia, sendo evidenciado em todos um aumento da taxa de captação de iodo e distribuição difusa do radiotraçador; $40(71,42 \%)$ pacientes foram submetidos à dosagem de TRAb, 37 destes com resultado positivo.

Dos 40 pacientes com BNT, $100 \%$ foram submetidos à ultrassonografia, que mostrou a presença de nódulos em todo o grupo, e o aumento de volume em 32 deles; 13 (32,5\%) submeteram-se à cintilografia ( 3 por BUNT e 10 com BMNT). Todos os BMNTs apresentaram padrões heterogêneos de distribuição do radiotraçador, com áreas de hipo e hipercaptação. Nos pacientes com BUNT, a cintilografia mostrou-se com uma área nodular única de hipercaptação, associada à supressão do restante do parênquima; em 8 (20\%) pacientes foram obtidas aferições do TRAb, todos com resultado negativo.

A Tabela 1 mostra a distribuição das variáveis epidemiológicas e de referência para os dois grupos. Observa-se diferença estatisticamente significativa na variável idade. A DG apresentou-se mais comum na faixa etária inferior a 50 anos $(66,1 \%)$, com média de idade de 43,9 anos, numa amplitude que varia de 23 a 78. Em contrapartida, os pacientes com BNT apresentaram-se em sua maioria com idade superior a 50 anos (92,5\%), e média de 65,8 anos, mínimo de 42 e máximo de 90 anos. Em ambas as etiologias, há predomínio de maior incidência no sexo feminino: $91,1 \%$ na DG e $87,5 \%$ no BNT.

A maioria dos pacientes, tanto os diagnosticados com DG $(80,5 \%)$ como os com BNT $(77,5 \%)$, vieram referenciados pela Unidade Básica de Saúde. O mesmo pode-se dizer com relação ao tratamento

Tabela 1 - Distribuição das variáveis epidemiológicas e de referência, para o total de pacientes com doença de Graves e bócio nodular tóxico $(n=96)$.

\begin{tabular}{|c|c|c|c|c|}
\hline \multirow[t]{2}{*}{ Variáveis } & \multirow[t]{2}{*}{ Total n (\%) } & \multirow{2}{*}{$\begin{array}{l}\text { Doença de Graves } \\
(n=56)\end{array}$} & \multirow{2}{*}{$\begin{array}{l}\text { Bócio Nodular } \\
\text { Tóxico }(n=40) \\
\text { n (\%) }\end{array}$} & \multirow[t]{2}{*}{$p$} \\
\hline & & & & \\
\hline \multicolumn{5}{|l|}{ Idade } \\
\hline Menos de 50 anos & $40(41,7)$ & $37(66,1)$ & $3(7,5)$ & $0,0^{*}$ \\
\hline 50 anos ou mais & $56(58,3)$ & $19(33,9)$ & $37(92,5)$ & \\
\hline \multicolumn{5}{|l|}{ Sexo } \\
\hline Feminino & $86(89,6)$ & $51(91,1)$ & $35(87,5)$ & $0,737 * *$ \\
\hline Masculino & $10(10,4)$ & $5(8,9)$ & $5(12,5)$ & \\
\hline \multicolumn{5}{|l|}{ Referência } \\
\hline Unidade Básica de Saúde & $76(79,2)$ & $45(80,5)$ & $31(77,5)$ & $0,734 *$ \\
\hline Outra & $20(20,8)$ & $11(19,5)$ & $9(22,5)$ & \\
\hline \multicolumn{5}{|l|}{ Manejo Inicial pela Referência } \\
\hline Tratamento com DAT\# & $58(60,4)$ & $37(66,1)$ & $21(52,5)$ & $0,18^{*}$ \\
\hline Não & $38(39,6)$ & $19(33,9)$ & $19(47,5)$ & \\
\hline
\end{tabular}


prévio com DAT: a maioria dos pacientes com DG $(66,1 \%)$ e BNT $(52,5 \%)$ já estava em tratamento com metimazol ou PTU em doses variadas antes da primeira consulta no ambulatório de Endocrinologia do HURCG.

A Tabela 2 descreve a apresentação dos sinais e sintomas nas duas formas de hipertireoidismo. $\mathrm{Na}$
DG, os sintomas mais encontrados foram: palpitação $(67,9 \%)$, perda de peso referida $(67,9 \%)$, tremores $(58,9 \%)$, nervosismo/ansiedade $(55,4 \%)$, fadiga/ astenia $(53,6 \%)$ e insônia $(48,2 \%)$. Nos pacientes com BNT, esses sintomas apresentaram-se com menor frequência. Comparado à DG, no BNT os sintomas compressivos foram mais frequentes,

Tabela 2 - Distribuição das variáveis "sinais e sintomas" para o total de pacientes com doença de Graves e bócio nodular tóxico $(n=96)$.

\begin{tabular}{|c|c|c|c|}
\hline \multirow[t]{2}{*}{ Variáveis } & \multirow[t]{2}{*}{$\begin{array}{c}\text { Total } \\
(n=96) \\
n(\%)\end{array}$} & \multirow{2}{*}{$\begin{array}{c}\text { Doença de Graves } \\
(\mathbf{n = 5 6 )} \\
\mathrm{n}(\%)\end{array}$} & \multirow{2}{*}{$\begin{array}{c}\text { Bócio } \\
\begin{array}{c}\text { Nodular Tóxico } \\
(\mathbf{n}=\mathbf{4 0})\end{array} \\
\mathrm{n}(\%)\end{array}$} \\
\hline & & & \\
\hline \multicolumn{4}{|l|}{ Sintomas da História Clínica } \\
\hline Palpitação & $48(50,0)$ & $38(67,9)$ & $10(25,0)$ \\
\hline Perda de peso referida & $50(52,1)$ & $38(67,9)$ & $12(30,0)$ \\
\hline Tremores & $38(39,6)$ & $33(58,9)$ & $5(12,5)$ \\
\hline Nervosismo/Ansiedade & $35(36,5)$ & $31(55,4)$ & $4(10,0)$ \\
\hline Fadiga/Astenia & $37(38,5)$ & $30(53,6)$ & $7(17,5)$ \\
\hline Insônia & $37(38,5)$ & $27(48,2)$ & $10(25,0)$ \\
\hline $\begin{array}{l}\text { Fragilidade de anexos } \\
\text { cutâneos }\end{array}$ & $32(33,3)$ & $25(44,6)$ & $7(17,5)$ \\
\hline Diarreia/Hiperdefecação & $17(17,7)$ & $15(26,8)$ & $2(5,0)$ \\
\hline Sudorese excessiva & $14(14,6)$ & $14(25,0)$ & 0 \\
\hline Dispneia & $28(29,2)$ & $13(23,2)$ & $15(37,5)$ \\
\hline Distúrbios menstruais & $11(11,5)$ & $11(19,6)$ & 0 \\
\hline Intolerância ao calor & $14(14,6)$ & $9(16,1)$ & $5(12,5)$ \\
\hline Hiporexia & $14(14,6)$ & $9(16,1)$ & $5(12,5)$ \\
\hline Disfagia & $22(22,9)$ & $7(12,5)$ & $15(37,5)$ \\
\hline Vertigem & $13(13,5)$ & $5(8,9)$ & $8(20,0)$ \\
\hline Dor retroesternal/Angina & $6(6,3)$ & $4(7,1)$ & $2(5,0)$ \\
\hline Disfonia & $7(7,3)$ & $4(7,1)$ & $3(7,5)$ \\
\hline Odinofagia & $2(2,1)$ & 0 & $2(5,0)$ \\
\hline Totalmente assintomático & $11(11,5)$ & $3(5,3)$ & $8(20,0)$ \\
\hline \multicolumn{4}{|l|}{ Sinais ao Exame Físico } \\
\hline Bócio & $80(83,3)$ & $46(82,1)$ & $34(85,0)$ \\
\hline Taquicardia & $38(39,6)$ & $27(48,2)$ & $11(27,5)$ \\
\hline Tremor fino de extremidade & $25(26,0)$ & $22(39,3)$ & $3(7,5)$ \\
\hline Oftalmopatia & $20(20,8)$ & $20(35,7)$ & 0 \\
\hline $\begin{array}{l}\text { Edema em membros } \\
\text { inferiores }\end{array}$ & $27(28,1)$ & $15(26,8)$ & $12(30,0)$ \\
\hline Dermopatia & $3(3,1)$ & $3(5,3)$ & 0 \\
\hline Fibrilação atrial & $6(6,3)$ & $3(5,3)$ & $3(7,5)$ \\
\hline Sinal de Pemberton & $4(4,2)$ & $1(1,8)$ & $3(7,5)$ \\
\hline $\begin{array}{l}\text { Nódulos palpáveis em } \\
\text { tireoide }\end{array}$ & $13(13,5)$ & 0 & $13(32,5)$ \\
\hline
\end{tabular}


incluindo dispneia $(37,5 \%)$, disfagia $(37,5 \%)$, disfonia $(7,5 \%)$ e odinofagia $(5 \%)$. Do total de 40 pacientes com BNT, oito (20\%) referiram ser totalmente assintomáticos. Na DG, apenas $5,3 \%$ dos pacientes apresentaram-se dessa forma.

Com relação aos sinais encontrados ao exame físico, o bócio foi o achado mais frequente, tanto na DG $(82,1 \%)$ quanto no BNT (85\%); sendo diferenciado pela presença de nodulações à palpação em $32,5 \%$ dos pacientes com BNT. Na DG, além do bócio, outros sinais frequentes foram: taquicardia $(48,2 \%)$, tremor fino de extremidades $(39,3 \%)$, oftalmopatia $(35,7 \%)$, edema de membros inferiores $(26,8 \%)$ e dermopatia $(5,3 \%)$. O sinal compressivo de Pemberton foi mais frequente no BNT $(7,5 \%)$, quando comparado à DG $(1,8 \%)$. Dermopatia e oftalmopatia não foram relatados no BNT.

A situação atual, o desfecho e o tratamento dos pacientes dos dois grupos estão apresentados na Tabela 3. Com relação aos pacientes com DG, 14,3\% encontravam-se em tratamento de hipertireoidismo há menos de 36 meses, todos em uso de metimazol, em doses que variavam de 10 a $60 \mathrm{mg} / \mathrm{dia}$. Em 13 $(23,2 \%)$ pacientes optou-se pela manutenção do uso de metimazol por mais de 36 meses, todos eles tendo obtido eutireoidismo com baixa dose de metimazol ( 5 a $10 \mathrm{mg} / \mathrm{dia}$ ). Terapias definitivas foram escolha em nove $(16,1 \%)$ pacientes, sendo sete por ablação com iodo radioativo e dois por tireoidectomia total. Destes, sete desenvolveram hipotireoidismo, em uso de doses variadas de levotiroxina, e um paciente manteve-se em hipertireoidismo após DTI ${ }^{131}$. Entraram em remissão após uso prévio de DAT, 13 $(23,2 \%)$ pacientes com DG. A taxa de abandono entre os pacientes com DG foi de $23,2 \%$.

Nos pacientes com BNT, três (7,5\%) estavam em tratamento com metimazol há menos de 36 meses e em nove pacientes (22,5\%) optou-se pela manutenção com metimazol em dose baixa por mais de 36 meses. Foram submetidos a terapias definitivas 12 pacientes $(30 \%)$, sendo sete por tireoidectomia total, quatro por tireoidectomia subtotal e um por $\mathrm{DTI}^{131}$. Desses pacientes que passaram por terapêuticas definitivas, dez evoluíram para hipotireoidismo e estavam em doses variáveis de levotiroxina. Dois pacientes não desenvolveram hipotireoidismo, ambos após tireoidectomia subtotal. A taxa de abandono do ambulatório nos pacientes com BNT foi de $40 \%$.

Tabela 3 - Descrição do desfecho e tratamento dos pacientes com doença de Graves e bócio nodular tóxico ( $n=96$ ).

\begin{tabular}{lccc}
\hline Variáveis & $\begin{array}{c}\text { Total } \\
(\mathbf{n = 9 6 )}\end{array}$ & $\begin{array}{c}\text { Doença de Graves } \\
\mathbf{n}(\mathbf{( \% )}\end{array}$ & $\begin{array}{c}\text { Bócio } \\
\text { Nodular Tóxico } \\
\mathbf{( n = 4 0 )}\end{array}$ \\
\cline { 2 - 3 } & $11(11,5)$ & $\mathbf{n}(\mathbf{\%})$ & $\mathbf{n}(\mathbf{\%})$ \\
$\begin{array}{l}\text { Uso de DAT*, em } \\
\text { hipertireoidismo, há menos } \\
\text { de 36 meses }\end{array}$ & $22(22,9)$ & $13(23,2)$ & $3(7,5)$ \\
$\begin{array}{l}\text { Uso de DAT*, em baixa } \\
\text { dose, há mais de 36 meses }\end{array}$ & $8(8,3)$ & $7(12,5)$ & $9(22,5)$ \\
$\begin{array}{l}\text { TD**, por Ablação com } \\
\text { Iodo Radioativo (DTI131) }\end{array}$ & $9(9,4)$ & $2(3,6)$ & $1(2,5)$ \\
$\begin{array}{l}\text { TD**, por Tireoidectomia } \\
\text { Total }\end{array}$ & $4(4,2)$ & 0 & $7(17,5)$ \\
$\begin{array}{l}\text { TD**, por Tireoidectomia } \\
\text { Subtotal } \\
\text { Remissão, após uso de }\end{array}$ & $13(13,5)$ & $13(23,2)$ & $4(10,0)$ \\
$\begin{array}{l}\text { DAT* } \\
\text { Abandono do } \\
\text { acompanhamento }\end{array}$ & $29(30,2)$ & $13(23,2)$ & - \\
\hline
\end{tabular}

*DAT: drogas antitireoidianas (metimazol ou propiltiouracil)

**TD: tratamento definitivo 
Do total de 96 pacientes com hipertireoidismo, $21(21,9 \%)$ foram submetidos a alguma modalidade de terapia definitiva. Desses, 18 (85,7\%) evoluíram com hipotireoidismo. Todos os pacientes submetidos à tireoidectomia total desenvolveram hipotireoidismo, necessitando de doses de levotiroxina de 150 a 200 $\mathrm{mcg} / \mathrm{dia}$. Nos pacientes que realizaram tireoidectomia subtotal, dois ( $50 \%$ ) desenvolveram hipotireoidismo, com reposição de $50 \mathrm{mcg} / \mathrm{dia}$ de levotiroxina. Dos oito pacientes submetidos à DTI ${ }^{131}$, sete evoluíram com hipotireoidismo, necessitando de reposição de levotiroxina em doses de 100 a $150 \mathrm{mcg} / \mathrm{dia}$.

Todos os pacientes foram submetidos a DAT em algum momento do tratamento. Inclusive quando se optou pelo tratamento definitivo, o uso de DAT foi utilizada como medida terapêutica inicial, mesmo que por um breve período, antes da tireoidectomia. Com relação à DAT de escolha no tratamento inicial, $82(85,5 \%)$ pacientes fizeram uso de metimazol, seis $(6,25 \%)$ de PTU e oito $(8,3 \%)$ fizeram o uso das duas drogas em momentos diferentes do acompanhamento. Para os pacientes submetidos a tratamentos prolongados (acima de 36 meses) com DAT, o metimazol foi a droga de escolha em todos os pacientes. Não foi relatado qualquer efeito adverso às medicações em 80 (83,3\%) pacientes. Os demais apresentaram algum tipo de efeito colateral inespecífico, como queixas gastrointestinais, cefaleia e edema. Todas as queixas surgiram nos primeiros 18 meses de uso da medicação. Não foram relatados efeitos mais severos, como agranulocitose e hepatotoxicidade.
Nas Tabelas 4 e 5 foram apresentadas a evolução dos dados vitais, antropométricos e laboratoriais dos pacientes com DG e BNT, respectivamente. Todas as variáveis expostas nas tabelas das duas etiologias apresentaram alterações estatisticamente significativas em relação aos valores na primeira e última consulta. Após o manejo no ambulatório, tanto os pacientes com DG quanto os com BNT apresentaram ganho de peso, redução da FC, aumento do TSH e reduções do T4 livre e T3 total. $O$ valor de TRAb foi avaliado nos pacientes com DG, com redução em relação à primeira consulta. A dose de metimazol mostrou-se menor na última consulta, tanto na DG quanto no BNT.

\section{DISCUSSÃO}

A etiologia mais prevalente de hipertireoidismo é a DG. No ambulatório em estudo, $58,3 \%$ dos casos apresentaram-se com essa patologia. Comparado a outras literaturas, o percentual de DG em pacientes com hipertireoidismo é variável, representando entre 50 a $80 \%$ dos $\operatorname{casos}^{3,8-9}$. Esta variação ocorre devido à heterogeneidade das populações estudadas, principalmente no que tange a fatores genéticos e nutricionais de aporte de iodo ${ }^{9}$. Em consonância com a literatura, encontramos o BMNT como segunda causa mais prevalente de hipertireoidismo ${ }^{10}$. Devese considerar que o estudo ocorreu em uma área de suficiência nutricional de iodo ${ }^{1}$.

Tabela 4 - Dados vitais, antropométricos e laboratoriais dos pacientes com doença de Graves na primeira e na última consulta $(n=56)$.

\begin{tabular}{|c|c|c|c|c|c|}
\hline \multirow[b]{2}{*}{ Variáveis } & \multicolumn{2}{|c|}{ Primeira Consulta } & \multicolumn{2}{|l|}{ Última Consulta } & \multirow[t]{2}{*}{$\mathbf{p}$} \\
\hline & Média (DP) & $\begin{array}{c}\text { Mediana } \\
(\text { Min-Max })\end{array}$ & Média (DP) & $\begin{array}{c}\text { Mediana } \\
\text { (Min-Max) }\end{array}$ & \\
\hline Peso & $62,9(13,8)$ & $61(35-112)$ & $68,9(15,1)$ & $66(44-121)$ & $<0,001 \#$ \\
\hline $\begin{array}{l}\text { Frequência } \\
\text { Cardíaca }\end{array}$ & $86,5(16,7)$ & $83(60-127)$ & $73,1(10,5)$ & $72(53-99)$ & $<0,001 \# \#$ \\
\hline TSH* & $0,242(0,74)$ & $0,01(0,001-3,44)$ & $1,76(2,92)$ & $0,96(0,005-16,74)$ & $<0,001 \#$ \\
\hline T4 L** & $3,3(2,0)$ & $3,0(0,66-10,1)$ & $1,5(1,1)$ & $1,19(0,57-7,77)$ & $<0,001 \#$ \\
\hline T3 T\& & $297,1(188,2)$ & $253(78-830)$ & $158,4(109,5)$ & $118,5(77-651)$ & $<0,001 \#$ \\
\hline TRAb\$ & $10,9(10,7)$ & $5,75(0,67-37,7)$ & $8,8(11,4)$ & $2,09(0,3-40)$ & $<0,01 \#$ \\
\hline $\begin{array}{l}\text { Dose de } \\
\text { Metimazol }\end{array}$ & $24,3(13,9)$ & $\begin{array}{c}20 \\
(5-80)\end{array}$ & $11,8(10,1)$ & $\begin{array}{c}10 \\
(5-60)\end{array}$ & $<0,001 \#$ \\
\hline
\end{tabular}

\#Teste de Wilcoxon; \#\#Teste $t$ de Student para amostras pareadas; ${ }^{*} \mathrm{TSH}=$ Hormônio Tireoestimulante (Tireotropina); ${ }^{* *} \mathrm{~T} 4 \mathrm{~L}=$ Tiroxina livre; \&T3T= Triiodotironina total; \$TRAb: Anticorpo Antirreceptor de TSH 
Tabela 5 - Dados vitais, antropométricos e laboratoriais dos pacientes com bócio nodular tóxico na primeira e na última consulta $(n=40)$.

\begin{tabular}{|c|c|c|c|c|c|}
\hline \multirow{2}{*}{ Variáveis } & \multicolumn{2}{|c|}{ Primeira Consulta } & \multicolumn{2}{|c|}{ Última Consulta } & \multirow[t]{2}{*}{$\boldsymbol{p}$} \\
\hline & Média (DP) & $\begin{array}{c}\text { Mediana } \\
(\text { Min-Max })\end{array}$ & Média (DP)* & $\begin{array}{c}\text { Mediana } \\
(\text { Min-Max) }\end{array}$ & \\
\hline $\begin{array}{l}\text { Frequência } \\
\text { Cardíaca }\end{array}$ & $85,4(17,9)$ & $\begin{array}{c}80 \\
(60-140)\end{array}$ & $76,0(12,3)$ & $\begin{array}{c}73,5 \\
(56-113)\end{array}$ & $0,001 \#$ \\
\hline Peso & $65,6(14,5)$ & $\begin{array}{c}65 \\
(40-100)\end{array}$ & $67,7(14,1)$ & $\begin{array}{c}68 \\
(37-95)\end{array}$ & $<0,01 \# \#$ \\
\hline TSH* & $0,6(0,9)$ & $\begin{array}{c}0,075 \\
(0,005-3,68)\end{array}$ & $8,8(23,1)$ & $\begin{array}{c}1,43 \\
(0,01-99,32)\end{array}$ & $<0,001 \#$ \\
\hline T4 L** & $1,6(0,7)$ & $\begin{array}{c}1,43 \\
(0,85-4,14)\end{array}$ & $1,1(0,3)$ & $\begin{array}{c}1,07 \\
(0,2-1,97)\end{array}$ & $<0,001 \#$ \\
\hline T3 T\& & $152,7(65,1)$ & $\begin{array}{c}141,5 \\
(86-388)\end{array}$ & $116,6(26,4)$ & $\begin{array}{c}112,5 \\
(80-171)\end{array}$ & $0,04 \#$ \\
\hline $\begin{array}{l}\text { Dose de } \\
\text { Metimazol }\end{array}$ & $13,0(11,6)$ & $\begin{array}{c}10 \\
(5-60)\end{array}$ & $10,4(9,4)$ & $\begin{array}{c}10 \\
(5-60)\end{array}$ & $0,02 \#$ \\
\hline
\end{tabular}

\#Teste de Wilcoxon ; \#\#Teste $t$ de Student para amostras pareadas; ${ }^{*} \mathrm{TSH}=$ Hormônio Tireoestimulante (Tireotropina); ${ }^{*} \mathrm{~T}$ 4L=Tiroxina livre; $\& \mathrm{~T} 3 \mathrm{~T}=$ Triiodotironina total

A maioria dos pacientes foi referenciada pela Atenção Básica (via Unidade Básica de Saúde), com manejo prévio com DAT: PTU ou metimazol. O Ministério da Saúde brasileiro orienta que todos os pacientes com hipertireoidismo tenham preferência no encaminhamento para a atenção especializada, com manejo inicial prévio pelos médicos generalistas ${ }^{11}$. No presente estudo, $60,4 \%$ dos pacientes chegaram ao ambulatório do HURCG sob manejo inicial com DAT. Hussain et al., em um estudo de coorte britânico de 2017, realizado com 633 pacientes com DG, observaram apenas $38,9 \%$ de manejo prévio com DAT por médicos de cuidados primários ${ }^{12}$.

No presente estudo, o sexo feminino apresentou maior prevalência de hipertireoidismo, independente da etiologia ser BNT $(87,5 \%)$ ou DG $(91,1 \%)$. Este dado condiz com trabalhos que indicam que a proporção de mulheres afetadas varia de quatro a dez vezes mais em relação ao sexo masculino ${ }^{12-13}$. A faixa etária apresenta-se de forma contrastante entre as duas etiologias, sendo um critério importante de diferenciação. O BNT, em especial o de característica multinodular, tem maior incidência na população com mais de 60 anos, quando comparado à DG, que se desenvolve principalmente na faixa etária dos 40 a 60 anos $^{12}$.

Na prática clínica ambulatorial, a determinação etiológica do hipertireoidismo é realizada pela análise conjunta de critérios clínicos, laboratoriais (TRAb) e de imagem (ultrassonografia, dopplerfluxometria e a captação de iodo na cintilografia) ${ }^{10}$. Não há um consenso, pelas diretrizes, sobre qual o melhor exame diagnóstico a ser associado à avaliação clínica. A Associação Europeia de Tireoide ${ }^{14}$ preconiza a associação entre a aferição do TRAb e o ultrassom. Por outro lado, a American Thyroid Association ${ }^{2}$ e o Consenso Brasileiro de Tireoide ${ }^{5}$ reconhecem o teste de captação de iodo radioativo na cintilografia como método útil na diferenciação etiológica. O Consenso Brasileiro ainda refere que a determinação sérica do TRAb é pouco necessária para o diagnóstico, quando a clínica sugere $D^{5}$.

A distinção entre DG e BNT pode ser realizada pela análise clínica dos sinais e sintomas. Na DG, a literatura infere que os pacientes comumente apresentam-se com a sintomatologia adrenérgica mais exuberante de hipertireoidismo ${ }^{8}$. Em consonância, os pacientes do ambulatório em estudo relataram, em sua maioria, nervosismo, sudorese 
excessiva, intolerância ao calor, palpitação, tremores, diarreia, fadiga, insônia e perda de peso. Ao exame físico, além dos sinais de tireotoxicose (taquicardia e tremor fino de extremidades), os pacientes com DG ainda possuem sinais patognomônicos da doença: a oftalmopatia e a dermopatia (mixedema pré-tibial), decorrentes do acúmulo sistêmico dos autoanticorpos $^{1}$. No estudo em questão, $35,7 \%$ dos pacientes com DG apresentaram oftalmopatia e $5,3 \%$ mixedema pré-tibial (dermopatia). Em outros estudos de revisão, encontrou-se percentagens que variaram de 25 a $60 \%$ para oftalmopatia e de um a $4 \%$ para a dermopatia ${ }^{3,15}$.

Nos pacientes com BNT, a sintomatologia clínica de tireotoxicose apresenta-se mais branda quando comparada à DG, entretanto, há maior propensão a desenvolver complicações cardiovasculares, como fibrilação atrial e insuficiência cardíaca $^{8}$. No estudo em questão, foi relatada dispneia (37,5\%) em maior proporção nos pacientes com BNT, o que talvez se associe com um maior grau de acometimento cardiovascular. Entretanto, há de se notar que os pacientes com BNT foram, em sua maioria, pacientes com mais de 50 anos. Estudos clássicos de insuficiência cardíaca demonstraram que idade por si só pode ser considerada um fator de risco para esta condição ${ }^{16}$.

Corroborando o caráter compressivo do bócio de etiologia nodular, que produz mais comumente sintomas de sensação globosa decorrentes da compressão esofágica e traqueal pelo bócio ${ }^{8}$, 0 presente estudo demonstrou maior quantidade de pacientes com disfagia $(27,5 \%)$ no grupo com BNT. O sinal compressivo de Pemberton (compressão do sistema venoso com a elevação dos braços, provocando falta de ar, distensão de veias cervicais e pletora facial ${ }^{17}$ ) foi evidenciado em $7,5 \%$ de dos nossos pacientes com BNT, sendo mais encontrado em idosos e pacientes com bócio volumoso de longa duração.

Na DG, a American Thyroid Association ${ }^{2}$ recomenda o uso da ablação com iodo radioativo $\left(\mathrm{DTI}^{131}\right)$ como primeira linha de tratamento. No Brasil, o Consenso Brasileiro de Tireoide ${ }^{5}$ orienta que seja realizado como primeira escolha um ciclo de 12 a 24 meses de DAT, preferencialmente metimazol. As terapias definitivas na DG seriam indicadas no hipertireoidismo persistente após uso desse primeiro ciclo de DAT, ou em pacientes que necessitem de um controle rápido e definitivo ${ }^{5}$. Neste estudo, somente $12,5 \%$ dos pacientes com DG foram submetidos à DTI ${ }^{131}$.

Torring et al., em um estudo randomizado, concluíram que as três formas de abordagem são igualmente eficazes como tratamento inicial da DG, entretanto as taxas de remissão da doença são menores nos pacientes tratados com DAT ${ }^{18}$. Em um primeiro ciclo de tratamento medicamentoso, a taxa de remissão, segundo a literatura, varia de acordo com a população, encontrando-se entre 30 a $50 \%{ }^{18-19}$. Neste estudo, a taxa de remissão foi de $23,2 \%$, considerando todos os pacientes. Cautela é necessária ao se avaliar este valor, já que mais de $20 \%$ de todos os pacientes com DG abandonaram o acompanhamento, podendo incorrer viés de seleção aos respondedores à DAT (os que abandonaram o tratamento podem tanto terem recorrido ao hipertireoidismo quanto atingido remissão).

Há uma tendência de mudança na terapia da DG, com aumento do tempo de uso de DAT. Estudos recentes sugerem que o tratamento com metimazol pode ser mantido por longos anos, se for obtido o eutireoidismo com baixa dose ( 5 a $10 \mathrm{mg})^{19-21}$. Lauberg et al. observaram que $90 \%$ dos pacientes com DG em tratamento com baixas doses de metimazol permaneceram continuamente eutireoidianos durante um acompanhamento médio de 6,7 anos ${ }^{21}$. Esta modalidade apresentaria menos complicações, mostrando-se uma opção segura e eficaz, quando comparada às terapias definitivas, que evoluem usualmente para estados de hipotireoidismo, necessitando de reposição contínua com levotiroxina ${ }^{5}$. A propensão ao uso prolongado de DAT pode ser observada neste estudo, onde $22,9 \%$ de toda a amostra válida e $23,2 \%$ dos pacientes com DG mantiveram o uso de metimazol em doses baixas por mais de 36 meses, pois alcançaram o eutireoidismo.

Os efeitos adversos mais graves do metimazol, como a agranulocitose, geralmente ocorrem durante os primeiros três a seis meses de tratamento e tendem a estar associados a altas doses, reforçando a seguridade dessa modalidade a longo prazo ${ }^{6}$. Em geral, a agranulocitose ocorre em cerca de 0,2$0,5 \%$ dos usuários de metimazol ${ }^{22}$, não tendo sido observada na amostra deste estudo.

Nos pacientes com BNT (seja BMNT ou BUNT), as terapias definitivas foram preferidas na maioria 
dos pacientes deste ambulatório, o que está de acordo com a Diretriz Americana ${ }^{2}$ e o Consenso Brasileiro de Tireoide $^{5}$, nos quais a tireoidectomia pode ser considerada a primeira escolha. A terapia com DAT por longos períodos seria indicada em pacientes com comorbidades e que não podem ser submetidos ao procedimento cirúrgico ${ }^{2,5}$. Azizi et al., em um estudo realizado em pacientes com BMNT, confirmaram que o tratamento com metimazol em dose baixa a longo prazo é tão seguro e eficaz quanto, não sendo inferior à terapia definitiva ${ }^{23}$. No presente estudo, $22,5 \%$ dos pacientes com BMNT estavam em uso de DAT por mais de 36 meses e mantiveram-se em eutireoidismo, mostrando-se certa tendência à manutenção do tratamento medicamentoso.

A resposta terapêutica tanto para DG quando para BNT, foi estatisticamente significativa para todas as variáveis analisadas (peso, FC, nível de TRAb, dose de DAT e níveis hormonais). É esperado melhora dos parâmetros após compensação do hipertireoidismo, bem como redução da dose dos usuários de DAT, ao longo do seguimento ${ }^{2,5}$.

Este estudo detém limitações, no que diz respeito à sua natureza transversal e por ter sido realizado em um único centro de estudos. Outro obstáculo foi que, ao basear-se na coleta secundária de dados, nem todas as variáveis se encontravam disponíveis. Além disso, o desfecho dos pacientes, analisado segundo o tratamento ou a remissão, pode ter sido sub ou superestimado pela alta taxa de abandono do ambulatório. Apesar das limitações, o trabalho apresentou importantes dados sobre a prática clínica da atenção especializada em hipertireoidismo, por um levantamento de aspectos clínicos e de tratamento desses pacientes acompanhados. Seus resultados poderão ajudar a estabelecer estratégias de gestão, de forma a auxiliar médicos e pacientes na tomada de decisões.

\section{CONCLUSÃO}

O hipertireoidismo mostra-se como uma doença heterogênea, desde a clínica inicial até a escolha terapêutica, entre suas etiologias mais prevalentes: a DG e o BNT. Os dados deste trabalho auxiliam a traçar um perfil clínico e observar a tendência de tratamento em pacientes com hipertireoidismo. Assim, atualmente verifica-se propensão a priorizar as terapias em longo prazo com baixas doses de metimazol, como foi evidenciado neste trabalho, reservando-se as terapias definitivas para os casos em que não haja controle medicamentoso eficiente ou no caso de uma doença muito severa.

\section{REFERÊNCIAS}

1. Kim M, Ladenson P. Tireoide. In: Goldman L, Ausinello DA. Goldman Cecil Medicina. 24 ed. Vol 2. Rio de Janeiro: Elsevier; 2014. p. 1665-80.

2. Ross DS, Burch HB, Cooper DS et al. 2016 American Thyroid Association Guidelines for Diagnosis and Management of Hyperthyroidism and Other Causes of Thyrotoxicosis. Thyroid. 2016; 26(10):1343-421. doi: 10.1089 /thy.2016.0229.

3. De Leo S, Lee SY, Braverman LE. Hyperthyroidism. Lancet. 2016; 388(10047):906-18. doi: http://dx.doi. org/10.1016/S0140-6736(16)00278-6

4. Graf $\mathrm{H}$. Manuseio do bócio uninodular e multinodular tóxico. In Vilar L, editor. Endocrinologia clínica. 6 ed. Rio de Janeiro: Guanabara Koogan; 2016. p. 327-34.

5. Maia AL, Scheffel RS, Meyer ELS, et al. Consenso Brasileiro de diagnóstico e tratamento do hipertireoidismo: recomendações do Departamento de Tireoide da Sociedade Brasileira de Endocrinologia e Metabologia. Arq Bras Endocrinol Metab. 2013; 57(3):205-32. doi: 10.1590/S0004-27302013000300006.

6. Burch HB, Cooper DS. Antithyroid drug therapy: 70 years later. Eur J of Endocrinol. 2018; 195(5):261-274. doi: http://doi.org/10.1530/EJE-18-0678

7. Yeo $\mathrm{CP}$, Khoo DH, Eng $\mathrm{PH}$, Tan HK, Yo SL, Jacob E. Prevalence of gestational thyrotoxicosis in Asian women evaluated in the 8 th to 14 th weeks of pregnancy: correlations with total and free beta human chorionic gonadotrophin. Clin Endocrinol (Oxf). 2001; 55(3):391-8. doi: $10.1046 / j .1365-$ 2265.2001.01353.x.

8. Brent GA. Clinical practice. Graves' disease. N Engl J Med. 2008; 358(24): 2594-605. doi.: 10.1056/ NEJMcp0801880.

9. Pontes AAN, Adan LF, Costa ADM, et al. Prevalência de Doenças da Tireoide em Uma Comunidade do Nordeste Brasileiro. Arq Bras Endocrinol Metab. 2002; 46(5):544-9. doi: http://dx.doi.org/10.1590/S000427302002000500008.

10. Doubleday AR, Sippel RS. Hyperthyroidism. Gland Surg. 2020; 9(1):124-135. doi:10.21037/gs.2019.11.01.

11. Brasil. Ministério da Saúde. Universidade Federal do Rio Grande do Sul. Protocolos de encaminhamento da atenção básica para atenção especializada v.1 Endocrinologia e Nefrologia. Brasília: Ministério da Saúde; 2015. Disponível em http://bvsms.saude. 
gov.br/bvs/publicacoes/protocolos_atencao_basica_ atencao_especializada_endocrinologia.pdf.

12. Hussain YS, Hookham JC, Allahabadia A, et al. Epidemiology, management and outcomes of Graves'disease - real life data. Endocrine. 2017; 56(3): 568-78. doi: 10.1007/s12020-017-1306-5.

13. Guevara-Linares $X$, Jasso-Huamán L, Ramírez-Vela $\mathrm{RM}$, et al. Características clínicas, demográficas y perfil tiroideo de los pacientes hospitalizados por hipertiroidismo en un hospital general. Rev Med Hered. 2015; 26(3):141-6. Disponível em: http://www.scielo. org.pe/scielo.php?script=sci_arttext\&pid=S1018$130 \times 2015000300002 \&$ lng $=$ es.

14. Kahaly GJ, Bartalena L, Hegedus L, et al. 2018 European Thyroid Association Guideline for the Management of Grave's Hyperthyroidism. Eur Thyroid J. $2018 ; 7(4): 167-86$. doi: 10.1159/000490384.

15. Sharma A, Stan MN. Thyrotoxicosis: Diagnosis and Management. Mayo Clin Proc. 2019; 94(6):1048-64. doi: https://doi.org/10.1016/j.mayocp.2018.10.011.

16. Yang $H$, Negishi $K$, Otahal $P$, Marwick TH. Clinical prediction of incident heart failure risk: a systematic review and meta-analysis. Open Heart. 2015; 2(1):e000222. doi: 10.1136/openhrt-2014-000222.

17. De Filippis EA, Sabet A, Sun MR, Garber JR. Pemberton's sign: explained nearly 70 years later. J Clin Endocrinol Metab. 2014; 99(6):1949-54. doi: 10.1210/jc.20134240.

18. Törring $O$, Tallstedt $L$, Wallin $G$, et al. Graves' hyperthyroidism: treatment with antithyroid drugs, surgery, or radioiodine - a prospective, randomized study. J Clin Endocrinol Metab 1996; 81(8):2986-93. doi.: https://doi.org/10.1210/jcem.81.8.8768863.

19. Villagelin D, Romaldini JH, Santos BR, et al. Outcomes in Relapsed Graves'Disease Patients Following Radioiodine or Prolonged Low Dose of Methimazole Treatment. Thyroid. 2015; 25(12):1282-90. doi: 10.1089/thy.2015.0195.

20. Azizi F, Ataie L, Hedayati M, et al. Effect of long-term continuous methimazole treatment of hyperthyroidism: comparison with radioiodine. Eur J Endocrinol. 2005; 152(5):695-701. doi: https://doi.org/10.1530/ eje.1.01904.

21. Lauberg, P, Berman DC, Andersen S, et al. Sustained Controlo of Graves' Hyperthyroidism During Long-Term Low-Dose Antithyroid Drug Therapy of Patients with Severe Graves' Orbitopathy. Thyroid. 2011; 21(9):9516. doi: 10.1089/thy.2011.0039.

22. Stumpf MAM, Schrut GCA, Ramthun M, Onuma $S$, Osternack HECG. Methimazole-induced agranulocytosis and sepsis: was thyroid storm present or just being mimicked? Acta Endocrinol (Buchar). 2019; 15(4):522525. doi: 10.4183/aeb.2019.522.

23. Azizi F, Taikyar MA, Madreseh E, et al. Treatment of toxic multinodular goiter: Comparason of radiodine and long-term methimazole treatment. Thyroid. 2019; 29(5):625-30. doi: 10.1089/thy.2018.0397. 


\section{Contribuições dos autores:}

RAP coleta de dados e redação do manuscrito

RSC coleta de dados e redação do manuscrito

GCAS revisão e correção da versão final

ACGCK revisão e correção da versão final

MAMS revisão e correção da versão final

Autor Correspondente:

Matheo Augusto Morandi Stumpf

matheoaugusto@hotmail.com

Editor:

Prof. Dr. Felipe Villela Gomes

Recebido: 13/11/2020

Aprovado: : 22/12/2020 